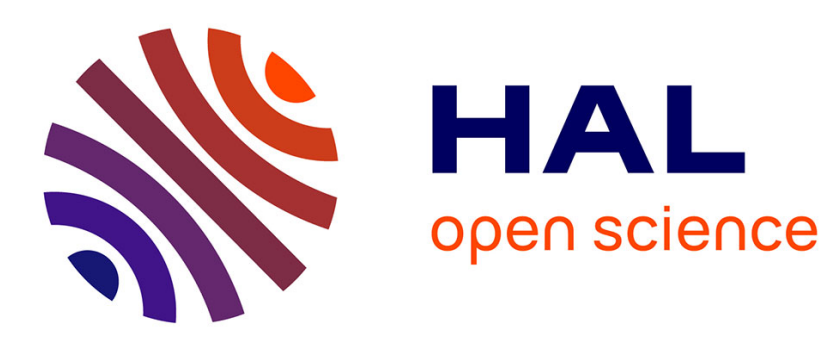

\title{
The slumping of a cohesive granular column: Continuum and discrete modeling
}

Anaïs Abramian, L. Staron, Pierre-Yves Lagrée

\section{To cite this version:}

Anaïs Abramian, L. Staron, Pierre-Yves Lagrée. The slumping of a cohesive granular column: Continuum and discrete modeling. Journal of Rheology, 2020, 64 (5), pp.1227-1235. 10.1122/8.0000049 . hal-03014980

\section{HAL Id: hal-03014980 \\ https://hal.science/hal-03014980}

Submitted on 19 Nov 2020

HAL is a multi-disciplinary open access archive for the deposit and dissemination of scientific research documents, whether they are published or not. The documents may come from teaching and research institutions in France or abroad, or from public or private research centers.
L'archive ouverte pluridisciplinaire HAL, est destinée au dépôt et à la diffusion de documents scientifiques de niveau recherche, publiés ou non, émanant des établissements d'enseignement et de recherche français ou étrangers, des laboratoires publics ou privés. 


\title{
The Slumping of a Cohesive Granular Column: Continuum and Discrete Modelling
}

\author{
Anais Abramian, ${ }^{1,}$ a) Lydie Staron, ${ }^{1}$ and Pierre-Yves Lagrée ${ }^{1}$ \\ Sorbonne Université, CNRS - UMR 7190, Institut Jean Le Rond d'Alembert, F-75005 Paris, \\ France
}

Cohesion forces strongly alter the flow properties of a granular material. To investigate this influence, we focus on a simple configuration: the collapse of a cohesive granular column. To do so, we adopt a numerical approach and implement a peculiar rheology in a Navier-Stokes solver (Basilisk) : the so-called $\mu(I)$-rheology, usually used for dry granular materials, supplemented by a yield stress for cohesion. With this approach, we recover the stability of the column, assuming the classical Mohr-Coulomb criterion for failure. We then compare this approach with a code based on Contact Dynamics, which implies forces at the grain scale: we recover as well the stability of the column. Furthermore, this comparison enables us to estimate the macroscopic yield stress based on the cohesive contacts between grains, which bridges the gap between continuous and discrete approaches of cohesive granular matter.

\section{INTRODUCTION}

Cohesion forces strongly alter the flow properties of a granular material. Instead of flowing homogeneously, grains aggregate and flow intermittently. In Nature, a loss of cohesion in soils can trigger catastrophic landslides ${ }^{2}$. In industrial processes, cohesion sometimes prevent materials, like gypsum or plaster, to flow properly. In the worst cases, it can clog and stop the flow during a process chain. Techniques have been devised to characterize these materials, and in particular determine their "flowability". Although these measurements can be useful to compare two powders or give qualitative properties of the material, they still lack of a physical base.

To this end, cohesive forces have been modeled at the grain scale, theoretically and numerically. These cohesion forces can be either Van der Waals forces ${ }^{1}$, electrostatic forces, or induced by capillary bridges ${ }^{13}$. However, it is not an easy task to link these properties to the macroscopic flow of an assembly, and in particular to the friction coefficient or the yield stress. Recently, Gans et al. ${ }^{5}$ elaborated a coating agent based on a polymer which enables them to get a stable and reproducible cohesive granular material. Doing so, they linked the force between two grains with the macroscopic rheology of the material.

In the wake of these results, we investigate, numerically, the link between the rheology of cohesive material and cohesive forces at the grain scale. To do so, we develop two numerical implementations, based on different approaches: a continuum approach based on the macroscopic, material scale, and a discrete approach based on the grain scale.

From the macroscopic point of view, we describe the material as a fluid of a peculiar rheology. In the first instance, the $\mu(I)$-rheology is a good candidate as it succesfully modelled the flow of dry granular materials ${ }^{16,27,29}$. According to the latter, the shear stress $\tau$ is related to the pressure $P$ through ${ }^{4,6,14}$

$$
|\tau|=\mu(I) P
$$

where $\mu(I)$ is the friction, which can involve the static friction

\footnotetext{
a)Electronic mail: anaisabramian@gmail.com
}

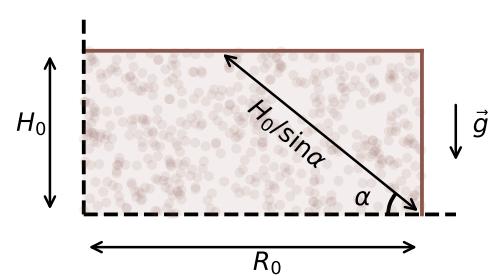

FIG. 1. 2-dimensional cohesive column of height $h$ in a gravity field. At equilibrium, friction and cohesion balances the weight of the upper corner along the surface of length $h / \sin \alpha$. This surface of incipient rupture forms an angle $\alpha$ with the horizontal.

coefficient $\mu_{s}$ as well as a complex dependence on the shear rate, encapsulated by the inertial number $I$.

To take into account cohesion in the material, we introduce a yield stress $\tau_{c}$ in the rheology, such that

$$
\tau=\tau_{c}+\mu(I) P
$$

and then solve the flow with a Navier-Stokes solver $(\text { Basilisk })^{16}$.

We compare these simulations with a code based on Contact Dynamics ${ }^{15,17}$, solving the motion of individual grains and giving access to individual grain-scale quantities, such as the forces between grains or the number of cohesive contacts.

In this article, we test our numerical implementations on a simple configuration: a granular column (Fig. 1). This is a challenging test because it covers a large range of flow regimes; meanwhile, its duration is short enough for the simulations. We expect the column to remain stable below a threshold height, due to the yield stress induced by cohesion. The column then flows when its initial height $H_{0}$ exceeds the threshold value:

$$
H_{y}=\frac{4 \ell_{c}}{\sqrt{\mu_{s}^{2}+1}-\mu_{s}},
$$

where $g$ is gravity, and $\ell_{c}$ is a cohesive length defined as:

$$
\ell_{c}=\frac{\tau_{c}}{\rho g}
$$


whith $\rho$ the density of the material.

Equation (3) is easily shown by considering the upper corner above the column, of mass $M=\rho h^{2} / 2 \tan \alpha$, sliding with friction and cohesion along a slope of angle $\alpha$. The MohrCoulomb failure criterion on the Mohr circle sets this angle of rupture $\alpha$ equal to ${ }^{30}$ :

$$
\alpha=\frac{\arctan \left(\mu_{s}\right)}{2}+\frac{\pi}{4},
$$

For example, with $\mu_{s}=0.3$, the column must exceed about $5 \ell_{c}$ to flow. Although this length controls the granular macroscopic cohesion from a continuum point of view, it is not well-defined at the grain scale. In the following, we attempt to bridge the gap by relating its value to the discrete contact forces.

To do so, we first test this threshold with the continuous approach (section II), and then the contact dynamics simulations (section III). We finally compare them and discuss about the relation between the material and the grain-scale cohesion (section IV).

\section{CONTINUOUS APPROACH}

\section{A. Rheology}

We describe here our continuous approach to model cohesion in the granular material. As mentioned in the introduction, we consider the granular column as a fluid of a peculiar rheology, namely a dry granular material supplemented by a yield stress (equation (2)).

For dry granular materials, the so-called $\mu(I)$-rheology is commonly used to model dense flows. This rheology takes into account the granular threshold to initiate the flow, and, involves a complex dependence on the shear rate, encapsulated by the inertial number ${ }^{6,7}$ :

$$
I=\frac{\dot{\gamma} d}{\sqrt{P / \rho}}
$$

We defined $\dot{\gamma}=\sqrt{2} D_{2}$ where $D_{2}=\sqrt{\mathbf{D}: \mathbf{D}}$ is the second invariant of the rate of strain tensor $\mathbf{D}, P$ the pressure, $d$ and $\rho$, the grains' diameter and density, respectively.

Then, the friction coefficient evolves as the following function of $I$ :

$$
\mu(I)=\mu_{s}+\frac{\Delta \mu}{I_{0} / I+1}
$$

where $\mu_{s}$ is the static friction coefficient, $\Delta \mu$ the difference between the dynamic and static friction coefficient and $I_{0}$ an initial inertial number. This rheology, first introduced by MiDi $(2004)^{6}$ for stationary flows, was shown to be valid in the intricate flow configuration of dry granular collapse by Lacaze \& Kerwell ${ }^{4}$ and implemented successfully in continuum models ${ }^{3,8,27,29}$ since Lagrée et al. $(2011)^{16}$.

Now, to account for the cohesion, we supplement this rheology by a yield stress $\tau_{c}$, which is classically used for viscoplastic fluids such as Bingham fluids. This threshold, on the

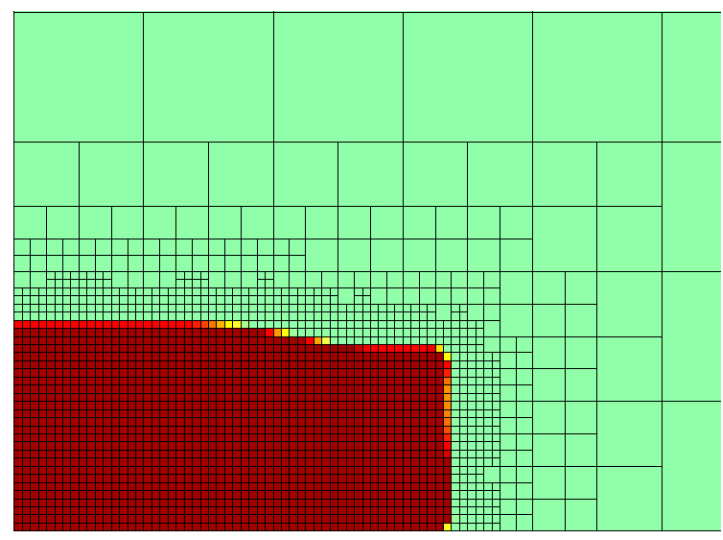

FIG. 2. Adaptive mesh refinement during a simulation of the collapse of a cohesive granular column (continuous approach). The color indicates the phase of the fluid. In red: cohesive granular material. In green: surrounding air.

contrary to the granular one, does not depend on the pressure. Overall, this peculiar rheology for our cohesive granular material translates in a relation between shear stress and pressure:

$$
|\tau|=\mu(I) P+\tau_{c}
$$

More generally, we assume that the internal stress tensor follows

$$
\sigma=-P \mathbf{I}+2 \eta \mathbf{D}, \text { with } \eta=\frac{\tau_{c}+\mu P}{\sqrt{2} D_{2}} .
$$

where $\eta$ is an effective viscosity.

This rheology assumes that frictional properties, described by $\mu(I)$ are independent of cohesive properties, encapsulated by $\tau_{c}$ only. This may not be the case for real cohesive material, where, for example, $\mu_{s}$ may vary with cohesion ${ }^{5}$. For lack of a comprehensive theory, we do not take this effect into account in the following.

In our simulations, we start by using the values of Jop et al. ${ }^{14}$ for the static coefficient $\mu_{s}=0.38, \Delta \mu=0.26$, and $I_{0}=0.279$. We also fix the grain size, such that we have 30 grains in a column radius $\left(d=R_{0} / 30\right.$, with $R_{0}$ the radius of the column). However, this grain size does not have a physical meaning in our description.

\section{B. Numerical implementation and parameters}

We now solve the Navier-Stokes equations for this fluid, using the flow solver Basilisk which is based on a projection method and a Volume-Of-Fluid approach ${ }^{11,16}$. Thus, we define two phases: the granular column and its surrounding air. The properties of the surrounding air does not affect the column dynamics provided that its viscosity and density are small enough compared to the column's ones ${ }^{16}$.

This solver uses an adaptive mesh refinement method ${ }^{12}$. Thus, we maximally refine the grid in the column and de- 


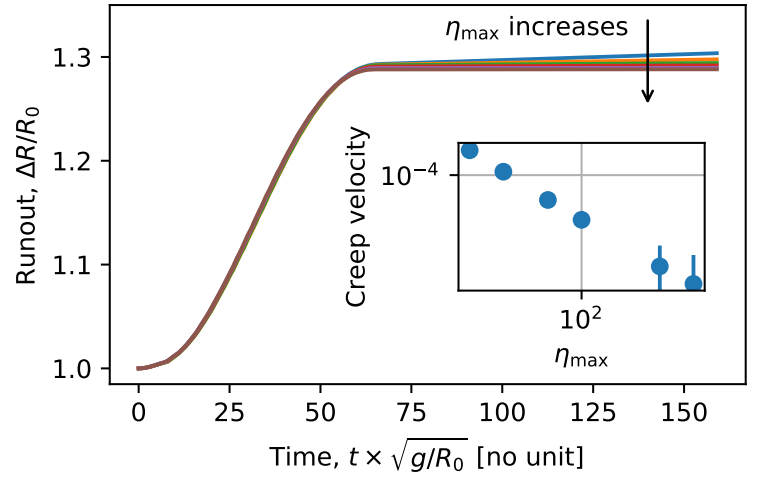

FIG. 3. Runout of the column as a function of time, for different regularization viscosity (continuous approach) $\eta_{\max }$ (with $\ell_{c} / H=0.1$ ). Inset. Final velocity of the material against the boundary viscosity $\eta_{\max }$.

\begin{tabular}{llll}
\hline \hline Length & Pressure & Velocity & Time \\
\hline$R_{0}$ & $\rho g R_{0}$ & $\sqrt{g R_{0}}$ & $\sqrt{R_{0} / g}$ \\
\hline \hline
\end{tabular}

TABLE I. Parameters used for the dimensional analysis.

crease its size in the surrounding fluid, which optimizes the time of computation (Fig. 2).

According to the rheology, the fluid then flows with an effective viscosity. However, as granular matter must stop when its viscosity diverges, we thus regularize its motion by introducing a maximum viscosity, $\eta_{\max }$. As a result, the column freezes and slowly creeps at the end of the simulation. We then have the following expression for the viscous stress and the the effective viscosity ${ }^{16}$ :

$$
\tau=2 \eta \mathbf{D}, \text { where } \eta=\min \left(\frac{\tau_{c}+\mu(I) P}{\sqrt{2} D_{2}}, \eta_{\max }\right) .
$$

There is not a unique manner to regularize the viscosity ${ }^{8,9}$, but we chose the simplest one by limiting the viscosity with a maximum, as did Lagree et al. ${ }^{16}$. They found that, for dry granular material, the values of $\eta_{\max }$ does not affect the final shape of the deposit as long as it is larger than 100.

We checked this dependency for our cohesive material by measuring the creeping velocity of the front, when it reached its final shape, and thus after typically $t=5 \sqrt{R_{0} / g}$. We observe that for $\eta_{\max }$ of above 100 , this variation remains smaller than about $10^{-5} R_{0}$, which we find to be negligible.

\section{Simulations}

We now show a first example for the slumping of a granular column with $\eta_{\max }=100$ (Fig. 4). In this example, we fix the domain size to $L=10$, and chose $H_{0}=1$ and $R_{0}=2$ for the initial geometry of the column. At its maximum, the grid size is then $L / 2^{8}$. We take the cohesive length equal to $\ell_{c} / H_{0}=0.1$, which remains small compared to the height of the column; the column thus releases and flows.

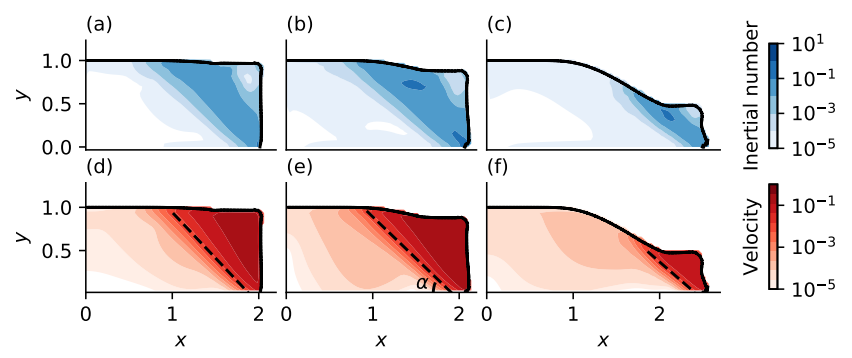

FIG. 4. Slumping of the cohesive granular column (continuous approach) with time. The colorbar indicates the intensity of the velocity. (a) $\&$ (d): $t=0$. (b) $\&$ (e): $t=1.5 \sqrt{R_{0} / g}$, (e) \& (f): $t=3 \sqrt{R_{0} / g}$ (final shape).

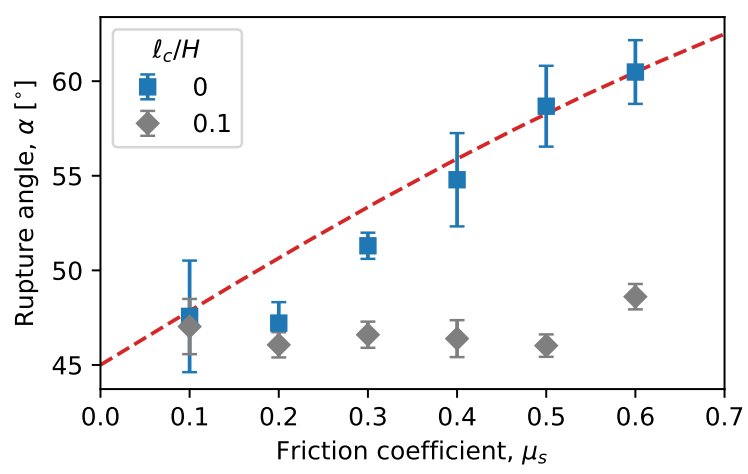

FIG. 5. Rupture angle as a function of the friction coefficient (continuous approach). Dashed red line: Mohr-Coulomb theory (equation (5)). Blue points: for granular columns without cohesion. Grey points: for cohesive columns, with a cohesion length of $\ell_{c} / H_{0}=0.1$.

By plotting the intensity of the inertial number $I$, we observe that a band appears, where the shear rate is maximum. In the corner above this band, the shear remains negligible, such that the corner does not deform and is almost undisturbed in the final shape. The final runout of the collapse, defined as the maximum distance travelled by the front of the flow, is then smaller than that of a cohesionless column.

This band makes an angle $\alpha$ with the horizontal. We expect that this angle is a function of the friction coefficient, through equation (5).

To measure this angle properly, we detect where the material moved from $10^{-2} \sqrt{g R_{0}}$ (dashed line, Fig. 4). Although this trend is well reproducible for a cohesionless granular material, it is not the case for a cohesive one (Fig. 5). In particular, theory systematically overestimates it. This may come from the uncertainty in the detection of the angle, which requires a local threshold criterion. Overall, as the trend is smooth, the angle has the good order of magnitude, and we will see that it does not affect the threshold of slumping, on which we focus in the following. 


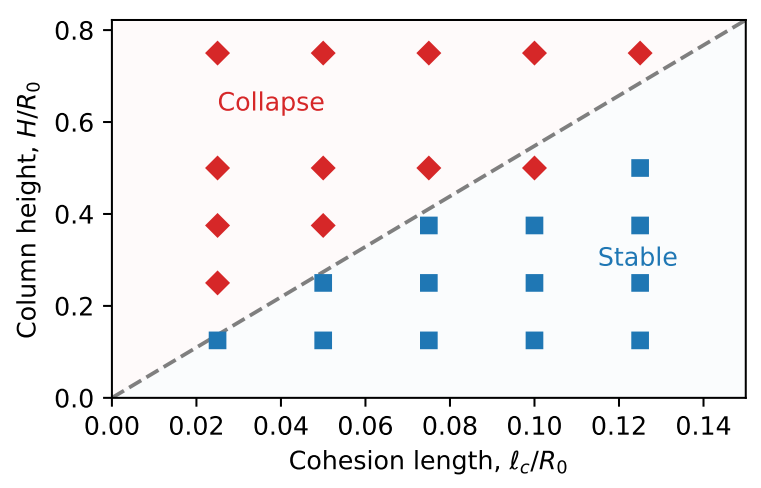

FIG. 6. Stability map. Grey dashed line: analytical limit (equation (3)). Points : numerical simulations with Basilisk (continuous approach). In blue: the pile is stable and never collapses. In red: the column collapses.

\section{Stability of the column}

We perform now a series of simulations where we vary the height of the column and the yield stress, and thus the cohesion length $\ell_{c}$.

First, we add a small cohesion $\left(\ell_{c}=0.1\right)$, and we vary the column's height (Vertical line in Fig. 6). If the column is high enough, it releases, then flows and acquires a stationary shape after approximately $t=5 \sqrt{R_{0} / g}$. Conversely, if the height is too small, the column does not flow and is cohesive enough to remain in its initial shape. This threshold follows equation (3) for a given cohesion.

This threshold depends on cohesion. Thus, we now keep a constant aspect ratio of the initial column (equal to 1), and we vary the dimensionless cohesion of the granular material $\ell_{c}$ (Horizontal line in Fig. 6). We recover the threshold in cohesion over which the column remains stable.

On the same figure, we plot the theoretical threshold given by equation (3), which provides a good agreement, without any fit parameter (dashed line, Fig. 6). This limit validates the implementation of cohesion in the granular column for small to moderate cohesion lengths.

We chose to rescale the height of the column and the cohesion length with the initial radius of the column $R_{0}$, that we did not vary from a simulation to another other. We also could use the cohesive length, or the grain diameter $d$, but the latter does not have a physical signification in this continuous approach; it is involved only in the $\mu(I)$-rheology, which does not evolve significantly with $d$. However, it will be better to do so when we compare our results with the discrete approach, which we do in the next section.

\section{DISCRETE APPROACH}

\section{A. Contact dynamics simulations}

Cohesive granular matter is simulated applying the Contact Dynamics (CD) algorithm ${ }^{15,17,19}$, already applied for col-

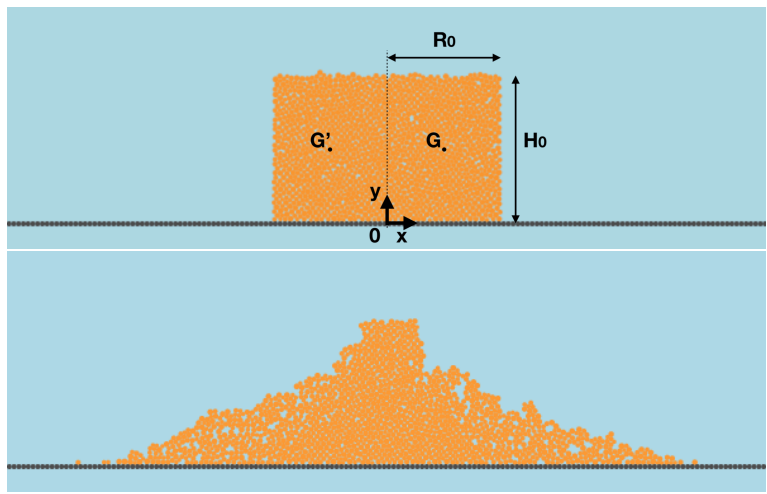

FIG. 7. An example of the discrete collapse of a cohesive column with the initial state (top picture) and the final state (bottom picture). The number of grains is 1500 , the $B_{\text {ond }}$ number is 100 .

umn collapse problems in ${ }^{16,25,26}$. The basic ingredients of this method are Coulombic solid friction and hardcore repulsion. Solid friction imposes locally that the normal contact force $f_{n}$ and tangential contact forces $f_{t}$ satisfy $f_{t} \leq \mu_{c} f_{n}$, where $\mu_{c}$ is the coefficient of friction at contact, while hardcore repulsion ensures that the values of normal forces computed are such that the overlap characterising contacts existence remains as small as possible. In the case of non-cohesive material, forces at contacts are exclusively compressive; in the cohesive case, forces in extension are allowed. This implies the introduction of a contact adhesive force $F_{a d h}$ that imposes the maximum value attainable by a force in extension before the contact is disrupted and opens. Based on the literature ${ }^{18,21}$, we set the value of the $F_{a d h}$ proportional to a granular Bond number and the mean weight of the grains involved in the contact.

$$
F_{a d h}=-B_{\text {ond }} m_{i j} g
$$

with $m_{i j}=\left(2 / m_{i}+2 / m_{j}\right)^{-1}$, and $i$ and $j$ are the two grains involved. Its value is systematically changed and its influence analysed in subsection IV.

The contact friction $\mu_{c}$ was set to 0.5 and its value/influence was not investigated nor discussed here. We may just make clear that the friction forces are computed for the normal forces without any cohesive contribution. Moreover, a coefficient of restitution $e$ set the amount of energy dissipated in collisions. Its value was set to $e=0.1$ (namely rather inelastic) and not varied.

Further technical details of the algorithm are not given in this paper, but may be found elsewhere ${ }^{22}$.

\section{B. Numerical set-up}

The systems simulated in this contribution are columns in 2D, as shown in Fig 7. The columns are made of circular beads of mean diameter $d=0.005 \mathrm{~m}$. uniformly distributed in the range [0.004, 0.006] to avoid geometrical ordering that may strongly dominate the failure and stability of the granular construction. The column are prepared by random rain in containers with initially a zero cohesion to ensure a volume 


\begin{tabular}{|c|c|c|c|c|c|}
\hline $\begin{array}{l}\text { Simulations } \\
\text { series }\end{array}$ & $\begin{array}{l}\text { Initial height } \\
H_{0}\end{array}$ & $\begin{array}{l}\text { Initial radius } \\
R_{0}\end{array}$ & $\begin{array}{l}\text { Initial radius } \\
a\end{array}$ & $\begin{array}{l}\text { Number of } \\
\text { grains }\end{array}$ & $\begin{array}{l}\text { Cohesion number } \\
B_{\text {ond }}\end{array}$ \\
\hline $\operatorname{simu} A(I)$ & $31 d$ & {$[10 d ; 42 d]$} & {$[0.72,3.06]$} & {$[580,2500]$} & {$[0,200]$} \\
\hline simu B (H) & {$[10 d, 46 d]$} & {$[10 d, 46 d]$} & $1.00 \pm 0.02$ & {$[186,4228]$} & {$[0,250]$} \\
\hline $\operatorname{simu} C(G)$ & {$[6 d, 43 d]$} & $16 d$ & {$[0.377,2.667]$} & {$[153,1326]$} & {$[0,200]$} \\
\hline
\end{tabular}

TABLE II. Table of discrete simulations performed.

fraction, or compacity, such as expected in a random packing with this size disparity; we obtain $\phi=0.85 \pm 0.03$. Once the packing has reached his final equilibrium state, a last series of computation time steps with $B_{\text {ond }} \neq 0$ are performed; this ensures that the initial condition for slumping (once the container walls are no longer there) is compatible with cohesion and the first few time steps of the computations do not see grains losing equilibrium because contact open before computation may restore their viability through cohesion. We should recall here that DC does not rely on an explicit formulation of the cohesive contact law using very small time steps, but on a non-smooth condition requiring going through an implicit iteration.

The dimension of the columns, namely initial height $H_{0}$ and initial radius $R_{0}$, are both changed. The intensity of the contact cohesive threshold is also systematically varied (through varying the $B_{\text {ond }}$ number, equation (14) in section IV). We essentially performed 3 series of simulations, with dimensions, number of grains, and cohesive threshold ( $B_{\text {ond }}$ number) all independently varied; a summary of the values adopted is given in table III B. Considering these values, the behaviour obtained in each series varies from collapse with spreading of most of the material, to slumping of part of the material following a well-defined failure zone, to equilibrium/stability with slow creeping. Figure 8 shows these three occurrences for a $B_{\text {ond }}$ number of successively 50, 100 and 150 .

\section{Evaluating the equilibrium of the discrete columns}

We need a criteria to decide whether a column is stable or unstable. Although this may sound an obvious thing to do, discrete columns do not always fall clearly into one category or the other, but might simply lose a small part of an edge, or see only few grains rolling down while the rest of the pile remains static, or see one angle perfectly static while the other loses equilibrium. To avoid to have to decide case by case who is stable and who is not (and then risk introducing subjective random criteria), we just quantify the slumping as the relative distance travelled by the secondary centres of mass $G$ and $G^{\prime}$ (i.e. centres of mass of each half of the initial column) following both axis, namely vertical slumping and horizontal

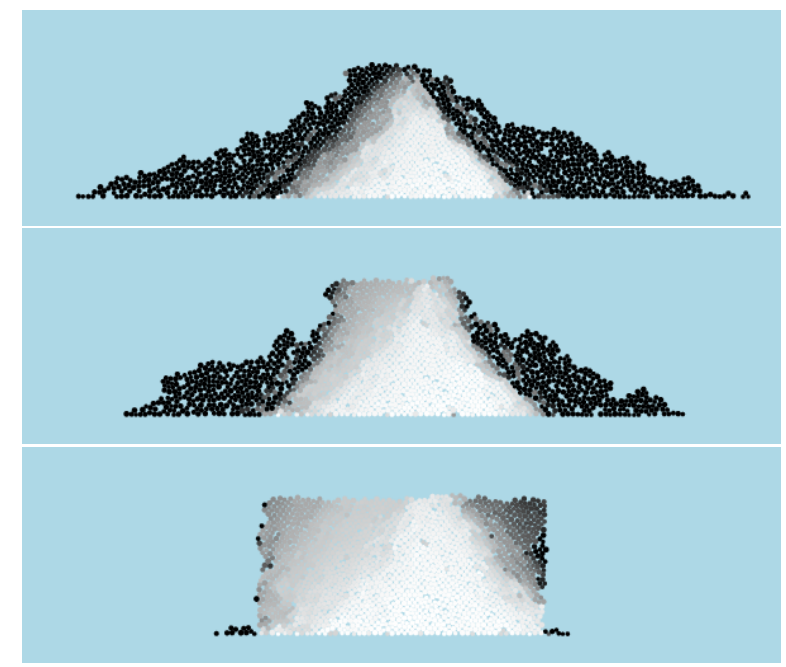

FIG. 8. An example of the final slumping of a discrete cohesive column with a $B_{\text {ond }}$ number of 50,100 and 150 (top to bottom). In black are particles whose total displacement is greater than $5 d$; the gray scale for the other grains is linear in cumulative displacement.

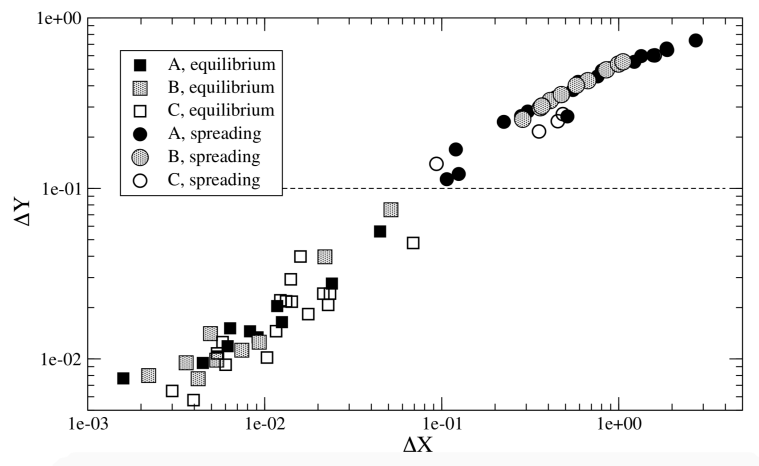

FIG. 9. Vertical slumping $\Delta Y$ against horizontal outspread $\Delta X$ for all discrete simulations. We chose the value $\Delta Y=0.1$ to distinguish columns at equilibrium ( $\square$ symbols) from those slumping $(\bigcirc$ symbols) although there are few simulations who do gather around this value. The gray scale are for the 3 series of simulations $\mathrm{A}, \mathrm{B}, \mathrm{C}$ presented in table III B. 


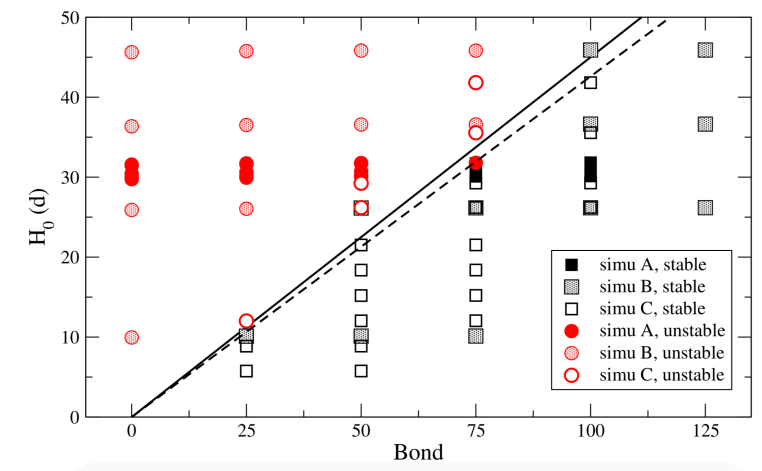

FIG. 10. Initial height $H_{0}$ (normalised by $d$ ) as a function of the $B_{\text {ond }}$ number for the 3 series of discrete simulations A, B, C presented in table III B. The full line which divides stable columns ( $\square$ symbols) and unstable ones ( $\bigcirc$ symbols) verifies $H_{0} / d=0.45 B_{\text {ond }}$. The dotted line shows the favorable case of the "Rumpf-Richefeu" prediction $^{20}$ (section IV C) with $\mu=0.3$ (equation (20)).

outspread, computing:

$$
\begin{aligned}
& \Delta X=\frac{\left|x_{(G, \infty)}-x_{(G, 0)}\right|}{\left|x_{(G, 0)}\right|}, \\
& \Delta Y=\frac{\left|y_{(G, \infty)}-y_{(G, 0)}\right|}{y_{(G, 0)}},
\end{aligned}
$$

where $x_{(G, 0)}$ and $x_{(G, \infty)}$, and $y_{(G, 0)}$ and $y_{(G, \infty)}$ (respectively $x_{\left(G^{\prime}, 0\right)}, x_{\left(G^{\prime}, \infty\right)}, y_{\left(G^{\prime}, 0\right)}$ and $\left.y_{\left(G^{\prime}, \infty\right)}\right)$, are the initial and final coordinates of the secondary centre of mass $G$ (respectively $G^{\prime}$ ). For each simulation, $\Delta X$ and $\Delta Y$ are computed for both secondary centres of mass $G$ and $G^{\prime}$ and then simply averaged. The vertical slump $\Delta Y$ is plotted as a function of the horizontal outspread $\Delta X$ in log-log coordinates for all simulations from table III B in Figure 9. We obtain two very distinct clouds of points for stable and unstable columns, joined by a handful of more uncertain realisations where only few grains roll down, creating a more ambiguous group closing the gap between two clear well-separated clouds of points. Nevertheless, these points are distinct enough to be distributed to one group or the other, so that we can now map out a stability graph plotting for each group, namely stable or unstable, the initial height $H_{0}$ (normalised by $d$ ) as a function of the $B_{\text {ond }}$ number, and compare it with the theoretical prediction (3), and with continuum simulations.

Using this criteria, we determine the behaviour (stable or not) of all simulations presented in table III B. The result is displayed in Figure 10, where two well-defined spaces for stable or unstable $\left(H_{0}, B_{\text {ond }}\right)$ pairs come out. From visual inspection, the linear dependence $H_{0} / d=0.45 B_{\text {ond }}$ forms a satisfactory boundary between both states, defining a discrete yielding height.

We can now compare the discrete granular failure behaviour with the continuous one.

\section{STABILITY ANALYSIS OF DISCRETE AND CONTINUUM COLUMNS}

\section{A. Comparing continuum and discrete approaches}

As reminded above, the stability of a continuum cohesive column of rheology $\tau=\tau_{c}+\mu(I) P$ requires that the height of the wedge remains below the yield value $H_{y}$ defined by equation (3). This threshold height, however, depends on the term $\tau_{c}$ referring to the macroscopic cohesion, or yield stress, valid over any representative volume of the continuum, at any rate valid for a whole column or wedge. No such quantity is readily available for the discrete counterpart of a cohesive column; indeed, the sole fully controlled ingredient in the discrete method is the cohesive contact force, namely hardly a quantity that one may identify with a "macroscopic cohesion". The effective macroscopic cohesion of a granular packing will of course reflect the value of contact cohesive forces at first order, but not only. The orientation of the contacts, the volume fraction of the system, and micro-textural aspect such as particle shape, will certainly play a role, as discussed in ${ }^{20,23}$. Meanwhile, determining computationally a quantity equivalent to $\tau_{c}$ would require a systematic analysis of the stress tensor for well defined configurations (such as shear cells), and this for steady flows, so that the friction could be unambiguously computed. This is hardly feasible in the transient complex dynamics studied here. Hence, in a first step, we will stick to the contact cohesion $F_{a d h}$, and discuss how to relate the $B_{\text {ond }}$ number to the macroscopic yield stress $\tau_{c}$

The definition of $F_{a d h}$ as used locally for each contact of the discrete systems in the CD simulations is given by:

$$
F_{a d h}=-B_{\text {ond }} m_{p} g
$$

In the following, we rather consider the mean contact adhesive force, replacing the weight of the two grains precisely in contact $m_{i j}$ by the mean particle weight $m_{p}=\pi d^{2} / 4, d$ being the mean grains diameter: In order to compare quantitatively the behaviour of discrete slumping collapses with their continuum counterparts, we a priori define a macroscopic cohesive stress representative for the granular packing simulated with the mean contact cohesive force $F_{a d h}$. Therefore, we introduce a characteristic length $L$ which we assume to be the macro/meso-scale over which cohesive stress applies:

$$
\frac{\left|F_{\text {adh }}\right|}{L}=\frac{B_{\text {ond }}}{L} \frac{\pi d^{2}}{4} \rho g,
$$

which, if we write $\tau_{c} \simeq F_{a d h} / L$, gives readily:

$$
\frac{\tau_{c}}{\rho g}=\frac{\pi d^{2}}{4 L} \times B_{\text {ond }} .
$$

The characteristic length $L$ is unknown; we may only guess that it probably is greater than a grain diameter (the smallest length scale in the system if we omit the explicit description of contacts), and of the order of few grains diameters by analogy with the often besought force chains. These are however, 


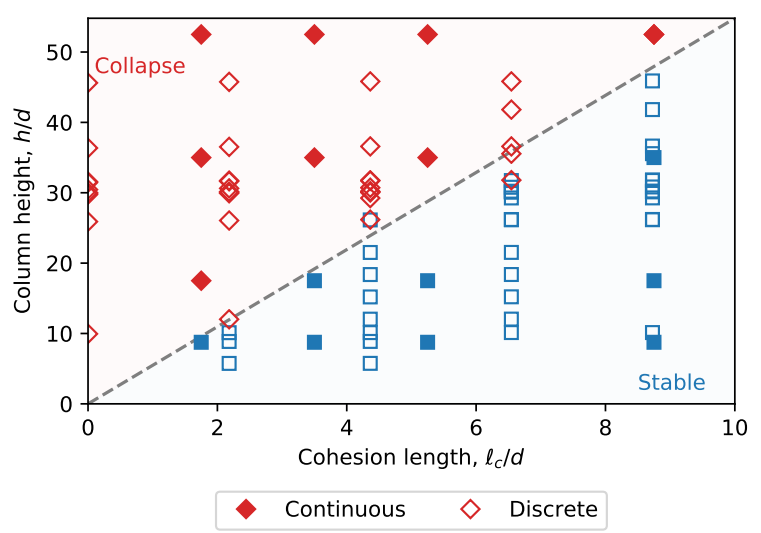

FIG. 11. Stability map with discrete and continuous data. Empty symbols: discrete simulations with Basilisk. Filled symbols: continuous simulations with Contact Dynamics. Grey dashed line: analytical limit (equation (3)). In blue: the pile is stable and never collapses. In red: collapse of the granular pile occurs.

if reasonable, yet only guesses, which ignore the fundamental role of the granular fabric when trying to model the mean mechanical properties of a granular sample. In the absence of a clear idea, we will merely assume that $L=n d$, where $n$ would be the typical number of grains onto which macroscopic cohesive stress builds up, and we will derive its value from the stability analysis of the simulated discrete columns shown in Figure 11.

The latter shows that the linear dependence $H_{0} / d=0.45 B_{\text {ond }}$ forms a satisfactory boundary between both states, defining a discrete yielding height that can be confronted with prediction (3). We can rewrite (3) using (16), so that:

$$
\frac{H_{y}}{d}=\frac{\pi}{\sqrt{\mu_{s}^{2}+1}-\mu_{s}} \times \frac{1}{n} \times B_{\text {ond }},
$$

where $\mu_{s}$ is the macroscopic friction, whose value for our systems is unknown. Considering that the contact friction is $\mu_{c}=0.5$, a reasonable estimate is $\mu_{s}=0.3^{24}$, which leads readily to $h_{y} / d=4.22 / n \times B_{\text {ond }}$, which gives $n=9$, namely $L=9 d$, which seems a reasonable value. Note that assuming $\mu_{s}=0.5$ leads to $L=11 d$, so that the result is poorly depending on the details of the friction coefficient (whose value remains generally bounded in a small interval ${ }^{24}$.

\section{B. Comparing discrete and continuum}

From the analysis above, we found that the stability analysis based on Mohr-Coulomb applies to discrete piles providing the cohesive stress is determined over a characteristic length of $L=9 d$. Injecting this result in equation (16) gives

$$
\frac{\ell_{c}}{d}=\frac{\pi}{36} B_{\text {ond }},
$$

which we can use to plot on a single graph both discrete and continuum data points for stable and unstable piles. Note that the grain diameter, in continuum simulations, plays a role only through the rheological model which accounts for the dependence on the inertial number $I$, inducing the frictional properties to vary with $d$. Verifying that this dependence is very small (since the $I$-dependence is virtually nil at the onset of the failure), we neglected it and varied $d$ in order to allow the normalised height $H / d$ for continuum piles to fall in the same interval as for discrete piles. Doing so, we can map discrete and continuum simulations onto a single final graph in Figure 11.

\section{Discussion}

Alternatively, we can adapt the analysis developed by Richefeu et $\mathrm{al}^{20}$ from Rumpf equation ${ }^{23}$ for 3D wet granular media, merely modifying it for 2D dry quasi-mono-disperse systems, and using expression (14) for contact cohesive forces $F_{a d h}$ rather than capillary forces. Following ${ }^{20}$, the density of cohesive contacts is given by half the mean number of cohesive contacts per particle $Z_{c}$ divided by the free volume in $2 \mathrm{D}$, i.e. the mean volume of a Voronoi cell surrounding the particle, i.e. the average particle volume $V_{p}=\pi d^{2} / 4$ divided by the solid fraction $\phi$. Considering the cohesive forces to be $F_{\text {adh }}=m g B_{\text {ond }}=\rho g V_{p} B_{\text {ond }}$, we obtain for the theoretical tensile strength for an assembly of cohesive particles:

$$
\sigma_{c}=\frac{d}{4} \phi Z_{c} \times \rho g \times B_{\text {ond }}
$$

with $\phi$ the packing volume fraction and $Z_{c}$ the mean number of cohesive contacts per particle. Hence, the theoretical cohesion $\tau_{c}=\mu_{s} \sigma_{c}$, gives readily:

$$
\frac{\tau_{c}}{\rho g}=\frac{d}{4} \mu_{s} \phi Z_{c} \times B_{\text {ond }} .
$$

Replacing theoretical expression (19) in the theoretical stability condition (3), we obtain:

$$
\frac{H_{y}}{d}=\frac{\mu_{s}}{\sqrt{\mu_{s}^{2}+1}-\mu_{s}} \times \phi Z_{c} \times B_{\text {ond }},
$$

which we can confront to the stability graph 10 .

We need therefore to evaluate $Z_{c}$; in Richefeu et $\mathrm{al}^{20}, Z_{c}$ (the number of capillary bounds in 3D) is assumed to be 6 . In our system, $Z_{c}$ is simply the mean number of contacts bearing negative forces per grain; its value for a $2 \mathrm{D}$ system counting 4228 grains with $B_{\text {ond }}=125$ is $Z_{c}=1.3$ (the mean number of contacts irrespective of the force transmitted is between 3 and 3.5). Taking $\phi=0.82$ (as measured), and assuming $\mu=0.3$, prediction (20) yields $h_{y} / d=0.426 B_{\text {ond }}$, in good agreement with what is observed from numerical simulations (graph 10). However, the result is sensitive to the value of $\mu$ chosen; taking $\mu=0.5$ leads to $h_{y} / d=0.86 B_{\text {ond }}$, in a much lesser agreement with observations.

More to the point, prediction (20) is very dependent on the value taken by $\phi$ and $Z_{c}$; a rapid inspection of the simulations shows that both quantities are dependent on the $B_{\text {ond }}$ number. For simulations taken from series B (table III B), we 
find that $\phi$ varies between 0.78 (for $B_{\text {ond }}=100$ ) and 0.82 (for $\left.B_{\text {ond }}=125\right)$, namely a modest $5 \%$ variations; but the mean number of cohesive contacts per particle varies between 0.75 (for $B_{\text {ond }}=50$ ) and 1.3 (for $B_{\text {ond }}=125$ ), creating alone a $40 \%$ uncertainty in expression (20) (see Figure 12). A dedicated work would be needed to clarify this aspect.

\section{CONCLUSION}

In this article, we investigated how cohesion influences the release of a granular column. We first developed a code where we solve Navier-Stokes equation with a VOF solver (Basilisk). We checked this code by investigating the threshold height over which a column flows or not. We found a good agreement with the Mohr-Coulomb criterion which predicts this threshold, without any fit parameter.

We then compared this approach with a code of Contact Dynamics, which models the dynamics of the column at the grain scale. By comparing the height threshold, we link cohesion forces at the grain scale with the macroscopic cohesive length, and thus the yield stress, as following:

$$
\frac{\ell_{c}}{d}=(0.087 \pm 0.005) B_{\text {ond }},
$$

for the given granular material simulated. This prefactor, however, depends on the grain-scale parameters used in our contact-dynamics algorithm, as the contact friction, or the grain-size distribution. Thus, we estimated this relationship in the light of the work of Rumpf-Richefeu, which provides an expression for the macroscopic cohesive length, as a function of the number of cohesive contacts, the compacity, and the friction coefficient:

$$
\frac{\ell_{c}}{d}=\frac{\mu_{s} \phi Z_{c}}{4} B_{\text {ond }} .
$$

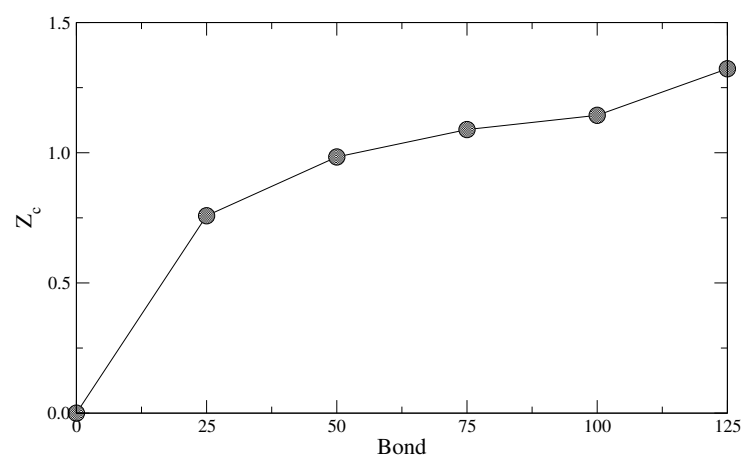

FIG. 12. Mean number of cohesive contacts (i.e. mean number of contacts bearing negative forces) per particle $Z_{c}$ as a function of the normalised cohesive force $B_{\text {ond }}=F_{a d h} / \mathrm{mg}$, for simulations from series B (table III B), with $R_{0}=H_{0}=46 d$, counting 4228 grains.
We measured $Z_{c}$ and $\phi$ in our contact dynamics simulations, and calculated the friction coefficient based on the contact friction coefficient. This expression yields thus a coefficient of $0.08 \pm 0.01$, which is in good agreement with our simulations. Still, the time step of computation could also have an influence in the discrete simulations, as shown by Abramian \& Staron $^{22}$.

Now that cohesion is numerically validated for the stability threshold of a column, much remains to be done to understand how cohesion alters the dynamics of the collapse, and the final shape of the deposit. To do so, we will likely need to compare numerics with experiments. Ideally, experiments would be conducted with a controlled-cohesive granular material, recently developed by Gans et al. ${ }^{5}$ This would provide new insights on the flow properties of a cohesive material and, hopefully, on the concept of "flowability".

\section{ACKNOWLEDGMENTS}

This work is part of the COPRINT project (http: //coprint226940055.wordpress.com) supported by the ANR grant ANR-17-CE08- 0017.

${ }^{1}$ Castellanos, A. (2005). The relationship between attractive interparticle forces and bulk behaviour in dry and uncharged fine powders. Advances in physics, 54(4), 263-376.

${ }^{2}$ Iverson, R. M. (2000). Landslide triggering by rain infiltration. Water resources research, 36(7), 1897-1910.

${ }^{3}$ Sachith Dunatunga, Ken Kamrin (2015). Continuum Modelling and Simulation of Granular Flows Throuh Their Many Phases, J. Fluid. Mech., 779, 483-513.

${ }^{4}$ Lacaze, L., \& Kerswell, R. R. (2009). Axisymmetric granular collapse: a transient 3D flow test of viscoplasticity. Physical Review Letters, 102(10), 108305 .

${ }^{5}$ Gans, A., Pouliquen, O., and Nicolas, M. (2019). A cohesion-controlled granular material. Submitted to Physical Review E.

${ }^{6}$ GDR MiDi 2004 (2004), On dense granular flows, Eur. Phys. J. E 14, 341 365

${ }^{7}$ Da Cruz, F., Emam, S., Prochnow, M., Roux, J. N., Chevoir, F. (2005). Rheophysics of dense granular materials: Discrete simulation of plane shear flows. Physical Review E, 72(2), 021309.

${ }^{8}$ Chauchat, J., Médale, M. (2014). A three-dimensional numerical model for dense granular flows based on the $\mu(I)$ rheology. Journal of Computational Physics, 256, 696-712.

${ }^{9}$ Frigaard, I. A., \& Nouar, C. (2005). On the usage of viscosity regularisation methods for visco-plastic fluid flow computation. Journal of non-newtonian fluid mechanics, 127(1), 1-26.

${ }^{10}$ Moreau, J.-J. (1994). Some numerical methods in Multibody Dynamics: Application to Granular Materials, European Journal of Mechanics, A/Solids 13-4, p.93-114.

${ }^{11}$ Scardovelli, R., \& Zaleski, S. (1999). Direct numerical simulation of freesurface and interfacial flow. Annual review of fluid mechanics, 31(1), 567603.

${ }^{12}$ Popinet, S. (2003). Gerris: a tree-based adaptive solver for the incompressible Euler equations in complex geometries. Journal of Computational Physics, 190(2), 572-600.

${ }^{13}$ Bocquet, L., Charlaix, E., \& Restagno, F. (2002). Physics of humid granular media. Comptes Rendus Physique, 3(2), 207-215.

${ }^{14}$ Jop, P., Forterre, Y., Pouliquen, O. (2006). A constitutive law for dense granular flows. Nature, 441(7094), 727.

${ }^{15} \mathrm{M}$. Jean and J.-J. Moreau, Unilaterality and dry friction in the dynamics of rigid bodies collections. In A. Curnier, (ed), Proc. of Contact Mech. Int. Symp., pp. 31-48. (1992) 
${ }^{16}$ Lagrée P.-Y., L. Staron \& S. Popinet, The granular column collapse as a continuum: validity of a two-dimensional Navier-Stokes model with the mu(I) rheology, J. Fluid Mech. vol 686, pp 378-408 (2011)

${ }^{17}$ J.-J. Moreau, Some numerical methods in Multibody Dynamics: Application to Granular Materials, European Journal of Mechanics, A/Solids 13-4, p.93-114 (1994)

${ }^{18}$ Nase, S. T., Vargas, W. L., Abatan, A. \& McCarthy, J. J. Discrete characterization tools for cohesive granular material. Powder Technol. 116, 214-223 (2001)

${ }^{19}$ Radjai F., M. Jean, J.-J. Moreau and S. Roux, Force distribution in dense two-dimensional granular systems, Phys. Rev. Lett., 77(2), 274 (1996)

${ }^{20}$ Richefeu V., MS El Youssoufi, F Radjai, Shear strength properties of wet granular materials, Physical Review E 73 (5), 051304 (2006)

${ }^{21}$ Rognon P. G. , J.-N. Roux, M. Naaïm and F. Chevoir, Dense flows of cohesive granular materials, J. Fluid Mech. vol. 596, pp. 21-47, doi:10.1017/S0022112007009329 (2008)

${ }^{22}$ Abramian A. \& Staron, L. (submitted), Time Steps v.s. Cohesion in NonSmooth Contact Dynamics Algorithm
${ }^{23}$ H. Rumpf, Zur Theorie der Zugfestigkeit von Agglomeraten bei Kraftubertragung an Kontaktpunkten, Chem.-Ing.-Tech. 42, 538 (1970)

${ }^{24}$ L. Staron, Instabilité des pentes granulaires, Institut de Physique du Globe de Paris (2002)

${ }^{25}$ Staron, L., \& Hinch, E. , Study of the collapse of granular columns using two-dimensional discrete-grain simulation. Journal of Fluid Mechanics, 545 , 1-27, doi:10.1017/S0022112005006415 (2005)

${ }^{26}$ Staron, L., \& Hinch, E. , The spreading of a granular mass: role of grain properties and initial conditions, Granular Matter, vol 9, p205-217, 2007

${ }^{27}$ L. Staron, P.-Y. Lagrée S. Popinet (2012) "The granular silo as a continuum plastic flow: the hour-glass vs the clepsydra" Phys. Fluids 24, 103301 (2012); doi: 10.1063/1.4757390

${ }^{28}$ L. Staron, P.-Y. Lagrée, S. Popinet (2014) "Continuum simulation of the discharge of the granular silo, A validation test for the $\mu(I)$ visco-plastic flow law" Eur. Phys. J. E (2014) 37: 5 DOI 10.1140/epje/i2014-14005-6 29

${ }^{30}$ Nedderman R. M., Statics and kinematics of granular materials, Cambridge University Press (1992) 